\title{
Event Based Sampling with Application to Vibration Analysis in Pneumatic Tires
}

\author{
Niclas Persson, Fredrik Gustafsson \\ Division of Communication Systems \\ Department of Electrical Engineering \\ Linköpings universitet, SE-581 83 Linköping, Sweden \\ WWW: http://www. comsys.isy.liu.se \\ Email: persson@isy.liu.se, fredrik@isy.liu.se
}

20th August 2001

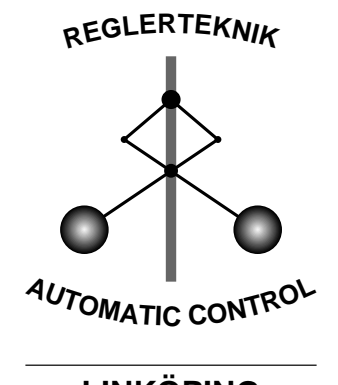

Report No.: LiTH-ISY-R-2374

Submitted to ICASSP'01, Salt Lake City, Utah, USA

Technical reports from the Communication Systems group in Linköping are available by anonymous ftp at the address ftp.control.isy.liu.se. This report is contained in the file 2374 .pdf. 


\begin{abstract}
Event based sampling occurs when the time instants are measured everytime the amplitude passes certain pre-defined levels. This is in contrast with classical signal processing where the amplitude is measured at regular time intervals. The signal processing problem is to separate the signal component from noise in both amplitude and time domains. Event based sampling occurs in a variety of applications. The purpose here is to explain the new types of signal processing problems that occur, and identify the need for processing in both the time and event domains. We focus on rotating axles, where amplitude disturbances are caused by vibrations and time disturbances from measurement equipment. As one application, we examine tire pressure monitoring in cars where suppression of time disturbance is of utmost importance.
\end{abstract}

Keywords: Event, Sampling, Tire, Pressure, Vibration 


\title{
EVENT BASED SAMPLING WITH APPLICATION TO VIBRATION ANALYSIS IN PNEUMATIC TIRES
}

\author{
Niclas Persson and Fredrik Gustafsson
}

\author{
Department of Electrical Engineering \\ Linköping University, SE-581 83 Linköping, Sweden \\ Email: $\{$ persson, fredrik\}@isy.liu.se
}

\begin{abstract}
Event based sampling occurs when the time instants are measured everytime the amplitude passes certain pre-defined levels. This is in contrast with classical signal processing where the amplitude is measured at regular time intervals. The signal processing problem is to separate the signal component from noise in both amplitude and time domains. Event based sampling occurs in a variety of applications. The purpose here is to explain the new types of signal processing problems that occur, and identify the need for processing in both the time and event domains. We focus on rotating axles, where amplitude disturbances are caused by vibrations and time disturbances from measurement equipment. As one application, we examine tire pressure monitoring in cars where suppression of time disturbance is of utmost importance.
\end{abstract}

\section{INTRODUCTION}

The classical sampling technique measures the amplitude of a signal $y(t)$ (continuous in both time and amplitude) at regular time intervals

$$
y[k]=y\left(k \Delta_{t}\right), \quad k=1,2, \ldots, N_{y} .
$$

The alternative studied here is to measure the times when the signal amplitude crosses pre-defined and here equidistant levels:

$$
t[k]=t\left(k \Delta_{y}\right), \quad k=1,2, \ldots, N_{t}
$$

This will be refered to as the event domain, while (1) is the usual time domain.

Figure 1 illustrates the principle. Compared to integral theory, these principles might be called Riemann and Lebesgue sampling, respectively. There are at least two reasons for studying event based sampled signals:

- Periodicity in the event domain comes naturally from certain disturbances and faults. Spectral analysis of $t[k]$ might reveal this information, which might leak out in the spectrum of $y[k]$.
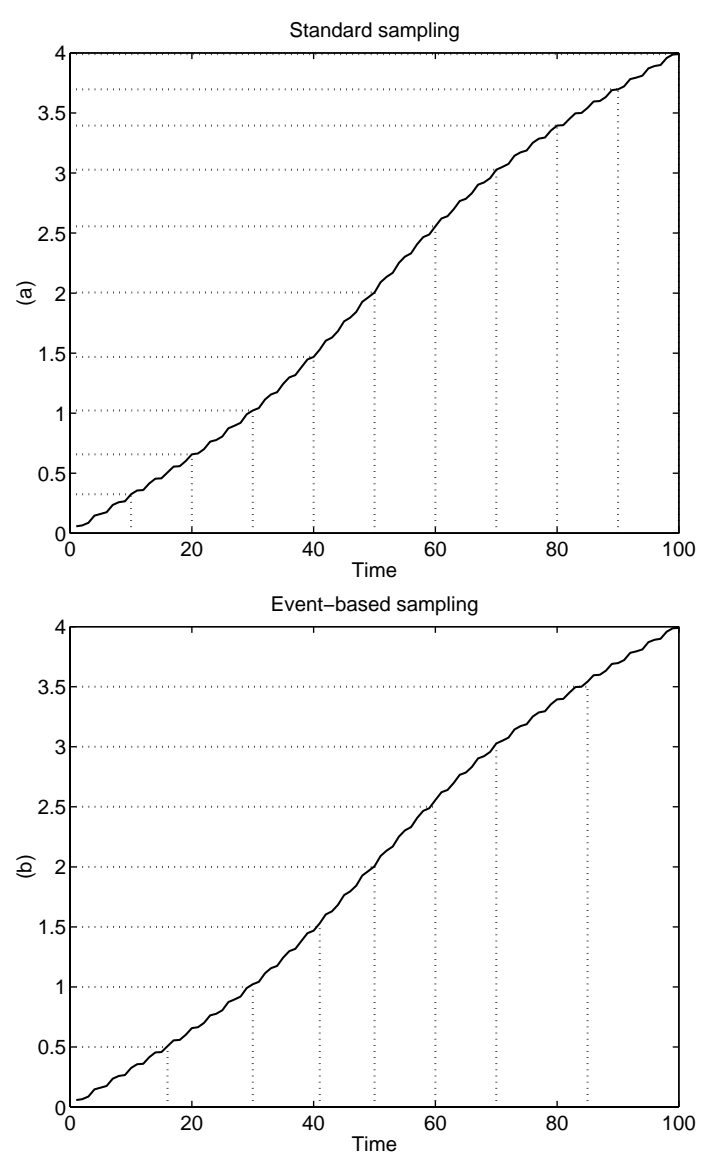

Fig. 1. Sampling with equidistant time (a) and amplitude (b) levels. 
- Many sensors deliver signals in the event domain.

We will focus on rotating axles as the wheel axles in cars, the camshaft axle in engines, motor axles in robots etc. In these applications, the standard sensor is a toothed wheel, see Figure 2. The sensor generates a pulse each time a tooth passes, either utilizing changes in the electromagnetic field or by using the Hall effect. Because of imperfect teeth, a periodic error will occur in the measurements.

Thus, the signal can be seen as consisting of three components

$$
y(t)=s(t)+e_{\text {amplitude }}(t)+e_{\text {time }}(t) .
$$

Here $s(t)$ is the signal component from angular velocity, $e_{\text {time }}(t)$ is a disturbance caused by the toothed wheel and also imbalance in the rotating parts, while $e_{\text {amplitude }}(t)$ is a disturbance on amplitude caused for instance by vibrations.

We will assume that we have a tool for interpolating back and forth between the time $y[k]$ and event $t[k]$ domains. The term $e_{\text {time }}(t)$ in (3) assumes that the event sampled signal is interpolated back to time domain data $y(t)$.

We suggest the following general definitions:

- A disturbance in the event domain is additive on $t[k]$ and gives rise to the term $e_{\text {time }}(t)$ in (3).

- A disturbance in the time domain is additive on $y[k]$ and is the (standard) noise term $e_{\text {amplitude }}(t)$ in (3).

In the application, the vibration contains useful information about the tire pressure, so the signal processing task is to extract $e_{\text {amplitude }}(t)$ while being insensitive to $e_{\text {time }}(t)$ and $s(t)$.

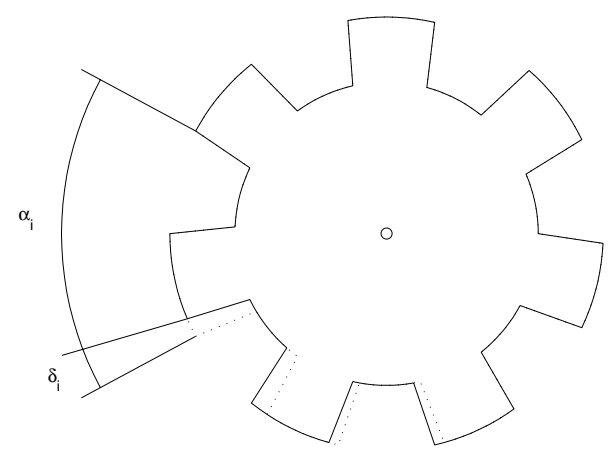

Ideal Toothed wheel Unideal Toothed Whee

Fig. 2. Toothed wheel performs event based sampling on rotating axles. The teeth are not exactly equally large, which implies a periodic measurement error.
In the signal processing community, event based sampling seems not very deeply studied. Existing literature on non-standard sampling focuses on multi-rate signal processing (the use of different sampling intervals), or nonuniform frequency resolution, see [1]. Recently, event-based sampling has been studied in the control community. The paper [2] examines if control actions only undertaken when the output passes certain levels can improve control performance. They analyse first order systems. As they point out, the analytic complexity seems tremendous for higher order systems.

That event based sampling is beneficial for modeling is pointed out in [3]. Modeling using a linear model may be able to describe measured data more accurately, when the time is replaced by the flow, which is supported by data from a paper plants.

\section{MOTIVATING EXAMPLE}

Consider an axle which is rotating with velocity

$$
v(t)=\underbrace{Y \frac{1}{\sqrt{2 \pi 3 T^{2} / 4}} e^{-\frac{(t-T / 2)^{2}}{3 T^{2} / 4}}}_{s(t)}+\underbrace{A \omega+A \sin (\omega t)}_{e_{\text {amplitude }}}
$$

where the latter term is an external harmonic disturbance.

The angle is thus given by $y(t)=\int_{-\infty}^{t} v(\tau) d \tau$. Sampling is performed $n=4$ times per revolution. There is a timing error in the sampling, so a vector $\varphi_{1}, \ldots, \varphi_{n}$ implies that

$$
t[k]=t\left(k \Delta_{y}+\varphi_{k} \bmod n\right) .
$$

Thus, the signal and sampling can be described as

$$
\begin{aligned}
y(t) & =Y \Phi\left(\frac{t-T / 2}{\sqrt{3 T^{2} / 4}}\right)+A \omega+A \omega \cos (\omega t), \\
t(y) & =y^{-1}(t) \\
t[k] & =t\left(k \Delta_{y}+\varphi_{k \bmod n}\right) .
\end{aligned}
$$

Here $\Phi$ is the Gaussian probability distribution function.

Figure 1 shows the result with

$A=1, \omega=2, Y=1, T=100, \Delta_{t}=1, \varphi=(0,1,-2,3)$.

Figure 3 shows frequency analysis of $|F F T(y[k])|$ (a,b) and $|F F T(t[k])|(\mathrm{c}, \mathrm{d})$, respectively, Figure 3(a) shows frequency analysis of $s(t)$ and (b) of $s(t)+e_{\text {amplitude }}(t)$. The amplitude disturbance gives rise to a distinct peak. Figure 3 (c) shows frequency analysis of $s(t)+e_{\text {amplitude }}(t)$ and (d) of $s(t)+e_{\text {amplitude }}(t)+e_{\text {time }}(t)$. The energy in the amplitude disturbance is leaking out and hard to see, and the visible peaks come from the time disturbance. If one interpolates the latter signal back to the time domain, the frequency 
content would be very much destroyed by the time disturbance, as the application study in the next section clearly demonstrates.
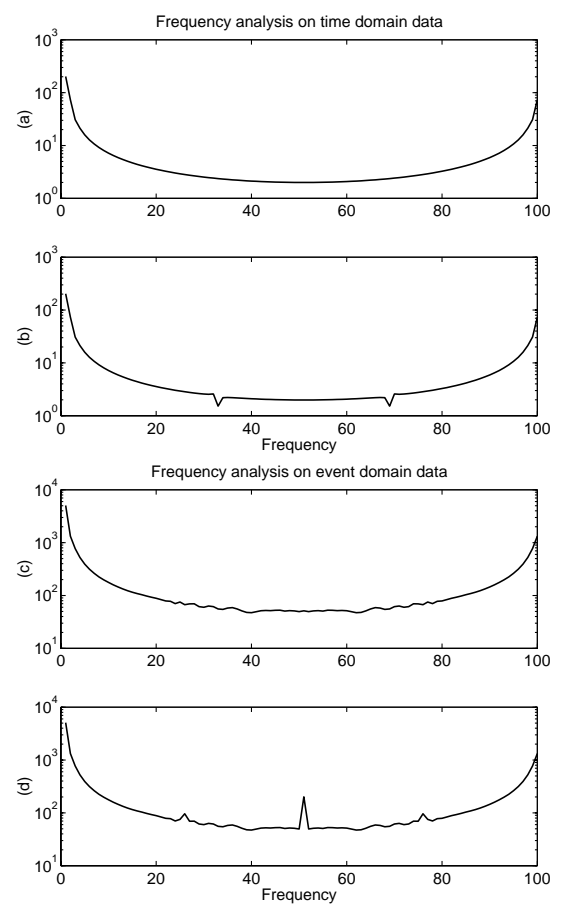

Fig. 3. Frequency analysis from time domain without disturbance (a) and with disturbance $s(t)+e_{\text {amplitude }}(t)(\mathrm{b})$. Frequency analysis from event domain with amplitude disturbance (c) and both kind of disturbances (d).

\section{TIRE PRESSURE MONITORING}

The wheel speed is central for many control tasks in modern cars, for instance ABS, traction control, anti-spin control, cruise control and dynamic stability control. Besides this, it is central for estimation purposes as navigation [4], friction estimation and tire pressure monitoring [5].

One approach to tire pressure monitoring is based on the assumption that the tire can be modeled as a spring-damper system, see [6, 7] and [8]. The spring constant then changes when the tire air pressure changes. A spring-damper system is characterized by its resonance frequency. The idea is to estimate this resonance frequency and detect whether it changes or not in order to detect e.g. a puncture before the tire breaks down so the vehicle can be stopped safely. The resonance frequency can be expected to be in the interval 40-50 Hertz, depending on tire type and pressure.

If the wheel speed signal is directly transformed to the frequency domain, Figure 4(a) shows that a disturbance is dominating. One of its harmonics lie in the interesting frequency range, so simple band-pass filtering is not enough.
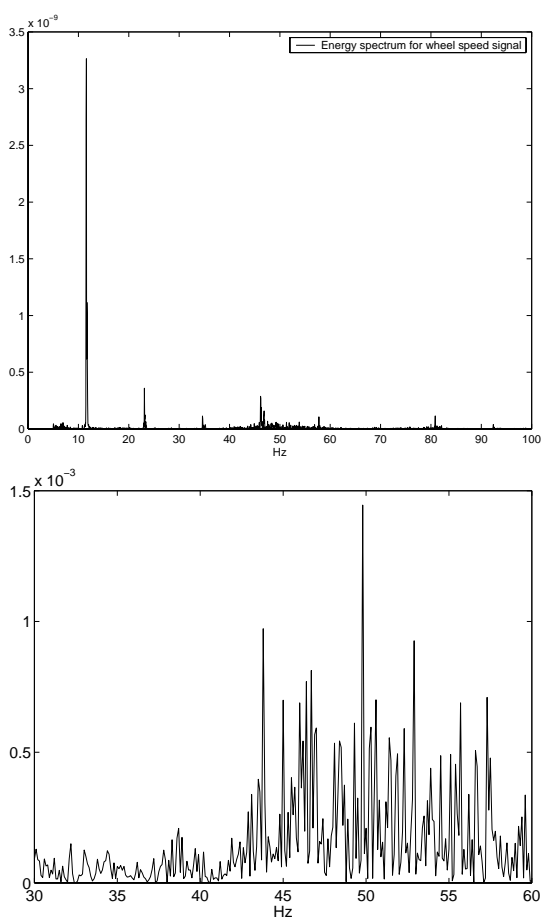

Fig. 4. Signal spectra from measurements (a) and after time disturbance estimation and rejection (b).

Returning to (3), the signal $s(t)$ has all its energy for low frequencies below $5 \mathrm{~Hz}$. Figure 4(a) shows the high pass original filtered signal to remove the otherwise dominating signal energy. The time disturbance $e_{\text {time }}(t)$ has its dominating disturbance frequency at $f=11 \mathrm{~Hz}$. Here the test drive was conducted with constant speed, that is why $e_{\text {time }}(t)$ gives a distinct peak. When car speed changes, its energy will leak out and the situation is even worse.

The proposal to get rid of the problems due to mechanical errors in the toothed wheel is to identify the magnitude of the errors. Because the toothed wheel can be subject of wear and tear or it can be hit by stones, the identification algorithm is recursive.

A linear regression model for the toothed wheel is

$$
\begin{aligned}
y[k] & =\omega[k] \cdot\left(t_{k}-t_{k-1}\right)-2 \pi / L \\
& =\varphi[k]^{T} \theta+e[k] \\
\theta & =\left(\delta_{1} \delta_{2} \cdots \delta_{L}\right)^{T} \\
\varphi[k] & =(01 \cdots 0) \quad \text { pos } k \quad \bmod L \text { is } 1 \\
\omega[k] & =\frac{2 \pi}{t_{k}-t_{k-L}} \\
\sum_{i=1}^{L} \delta_{i} & =0
\end{aligned}
$$

where $\omega$ is the angular velocity of the wheel and $L$ is the number of teeth in the toothed wheel. The measurement is 
here based on the mean velocity over one revolution of the wheel, and the deviation from the mean level is modeled as tooth offsets. An important assumption for (9) to work satisfactory is that the angular velocity is almost constant during one cycle (one wheel revolution), i.e. the time constant of the signal needs to be much larger than the cycle time. In vehicle dynamic applications, this is not a limitation because the time constant for the velocity of a car is much larger than one wheel revolution. An identification of the mechanical errors in a toothed wheel using (9) can be seen in Figure (5).

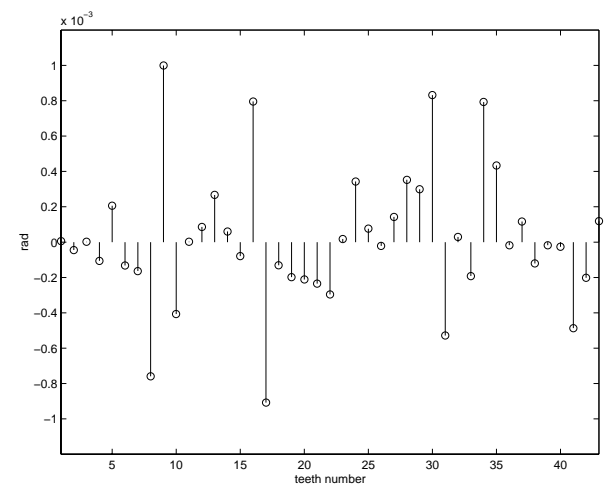

Fig. 5. Identified mechanical errors in toothed wheel using (9).

As can be seen in the figure some of the mechanical errors are too large to be neglected. Instead the identified offsets can be used to correct the original signal. The idea with the correction is to compensate for the mechanical errors:

$$
\Delta t_{c o r r}=T_{k}-\delta / \omega[k]
$$

After correction, the signal can be transformed, and the result can be seen in Figure 4(b). Obviously, (9) and (15) works and no signs from false frequencies from the mechanical errors can be seen in the figure. Now it is possible to identify the resonance frequency in the range $40-50 \mathrm{~Hz}$ corresponding to the tire air pressure. The details of this algorithm, not specific for event based sampling, are described in [6].

\section{CONCLUSIONS}

Event based sampling seems to be an important concept for noise rejection and estimation in rotating axles. It occurs naturally by the commonly used toothed wheel sensoring angular speed, but also in other type of flow sensors. It was pointed out by both a simulated example and real data from a car wheel that additive harmonic signals and timing errors in the sensor give rise to two totally different kind of disturbances, which may be analysed and rejected in the time and event domains, respectively. Finally, a method to estimate the timing errors was given and applied to real data for the purpose of tire pressure monitoring.

\section{REFERENCES}

[1] S. Bagachi and S.K. Mitra, The nonunifrom discrete Fourier transform and its applications in signal processing, Kluwer Academic Publishers, 1999.

[2] B. Bernhardsson and K-J Åström, “Comparison of periodic and event based sampling for first-order stochastic systems," in Preprints of the 14th IFAC World Congress, 1999.

[3] T. Andersson and P. Pucar, "Estimation of residence time in continuous flow systems with dynamics," Journal of Process Control, , no. 5, pp. 9-17, 1995.

[4] F. Gustafsson, S. Ahlqvist, U. Forssell, and N. Persson, "Sensor fusion for accurate computation of yaw rate and absolute velocity," in Submitted to SAE 2001, Detroit., 2001.

[5] F. Gustafsson, M. Drevö, U. Forssell, M. Löfgren, N. Persson, and H. Quicklund, "Virtual sensors of tire pressure and road friction," in Submitted to SAE 2001, Detroit., 2001.

[6] F. Gustafsson, M. Drevö, and N. Persson, "Tire pressure computation system," Swedish patent application nr 0002213-7, 2000.

[7] EP700798, “Tire pneumatic pressure detector," Equivalents US 5606122, 1996, Nippon Denso Co, Nippon Soken.

[8] J.Y. Wong, Theory of ground vehicles, John Wiley \& Sons, Inc, 2nd edition, 1993. 